Portland State University

PDXScholar

Electrical and Computer Engineering Faculty

Publications and Presentations

$11-1-1975$

\title{
Mode properties of annular gain lasers
}

Lee W. Casperson

Portland State University

Mohammad Shabbir Shekhani

Follow this and additional works at: https://pdxscholar.library.pdx.edu/ece_fac

Part of the Electrical and Computer Engineering Commons

Let us know how access to this document benefits you.

\section{Citation Details}

Lee W. Casperson and M. Shabbir Shekhani, "Mode properties of annular gain lasers," Appl. Opt. 14, 2653-2661 (1975).

This Article is brought to you for free and open access. It has been accepted for inclusion in Electrical and Computer Engineering Faculty Publications and Presentations by an authorized administrator of PDXScholar. Please contact us if we can make this document more accessible: pdxscholar@pdx.edu. 


\title{
Mode properties of annular gain lasers
}

\author{
Lee W. Casperson and M. Shabbir Shekhani
}

\begin{abstract}
The properties of a new class of laser resonators are investigated theoretically and experimentally. In these lasers the radiation propagates longitudinally in an annular amplifying medium, and useful low loss modes can be obtained even when the axial region is obscured. Alignment characteristics and far field patterns are discussed, and experiments have been conducted using coaxial double-discharge $\mathrm{CO}_{2}$ devices.
\end{abstract}

\section{Introduction}

The mode properties of ordinary spherical-mirror Fabry-Perot lasers have been well understood for many years. In most practical lasers the modes can be described in terms of familiar Laguerre-Gaussian ${ }^{1}$ and Hermite-Gaussian ${ }^{2}$ functions. A feature common to all Fabry-Perot type lasers is that the energy is confined fairly close to the $z$ axis. Recently, however, lasers are being developed that have entirely different gain distributions and mode characteristics. The situation of particular interest here involves a gain medium occupying an annular region of space. $^{3-7}$ Along the axis of the laser is an obstacle that is opaque to the laser radiation. In such a laser the radiation is excluded from the central region, and conventional Laguerre-Gaussian and Hermite-Gaussian modes are not appropriate.

There are a variety of circumstances where annular gain media occur. For example, flashlamp pumped dye lasers can be operated with the flashlamp situated along the laser axis. ${ }^{5}$ This configuration provides efficient energy coupling. The surrounding dye yields an annular amplifying region and can also provide cooling for the lamp. The same considerations are involved in solid flashlamp pumped lasers, and a tube-shaped Nd:glass laser has been demonstrated. ${ }^{6}$ The resonators for these lasers must provide low diffraction loss ring-shaped modes, since the axes of the resonators are blocked.

Similar mode problems arise in some of the newer types of gas lasers. The earliest gas lasers were all longitudinally pumped, but more recently various coaxial discharge configurations have been employed.

The author is with the School of Engineering \& Applied Science, University of California, Los Angeles, California 90024.

Received 2 May 1975.
In a $\mathrm{CO}_{2} \mathrm{TE}$ device, for example, coaxial preionization has been achieved by means of an auxiliary radial electron beam. ${ }^{7}$ In, that laser spherical mirrors were used, which precluded fundamental mode operation. For most practical applications the high order modes have undesirable focusing and propagation behavior. In the work described here, we have developed a new type of resonator using aspheric mirrors for improved mode control.

Several advantages of the coaxial discharge design will become apparent. The laser can be built into a grounded metal pipe. This provides compactness, ruggedness, and safety characteristics that cannot be approached by glass or ceramic longitudinally pumped lasers, and the symmetry of the current distribution reduces the likelihood of arcing. Comparatively low voltages can be used, and heat conduction through the walls is improved. With $\mathrm{CO}_{2}$ lasers, for example, low temperatures are essential to prevent thermal population of the lower laser level. Annular amplifying media also occur in some new types of high-powered chemical lasers involving HF and DF. In these lasers the reacting chemicals are mixed and propagated radially through the mode volume, and the axial region is obscured by gas mixing hardware.

There are various ways that one can approach the problem of resonator development for annular gain lasers. The basic configuration is shown in Fig. 1. The laser radiation travels longitudinally in the amplifying annulus between two parallel mirrors. The earliest nonplane designs used mirrors that in cross section have circular curvature centered away from the laser axis, ${ }^{3}$ and the advantages of such designs can be readily appreciated. For an arbitrary sized obstruction along the laser axis, mirrors can be chosen that localize the laser radiation outside of the axial region. The resulting output modes diffract slowly and may have no node lines across the phase fronts. In the following sections, the earlier mode studies are generalized to include the interaction with radially inhomogeneous amplifying and refract- 


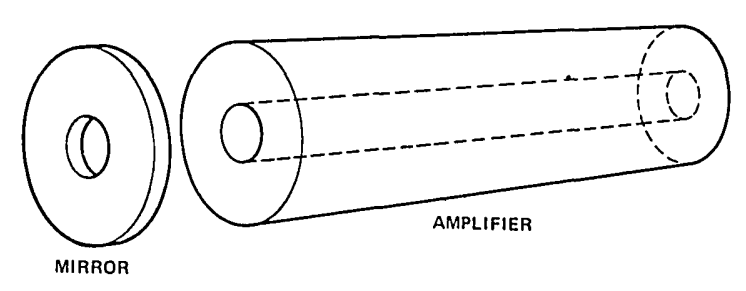

Fig. 1. Schematic drawing of an annular gain laser.

ing media. Such media occur commonly in practice, and the resulting beam distortion can be compensated by the mirror design. It is shown that the leading correction for azimuthal field variations has the same form as that obtained with a radially varying index of refraction. The radial field variations are characterized by Hermite-Gaussian functions, and misalignment of the mirrors causes the lasing to occur on only one side of the resonator. Our experiments were conducted using coaxial double-discharge $\mathrm{CO}_{2}$ lasers. Except for the construction differences and ringshaped beam modes, these devices are found to be similar in behavior to conventional TEA lasers.

\section{Beam Modes of Complex Annular Media}

The vector electromagnetic beam modes in a general inhomogeneous medium are governed by the equations $^{8}$

$$
\begin{aligned}
& \nabla \times \nabla \times \bar{E}-\omega^{2} \mu \epsilon \bar{E}=(\nabla \mu / \mu) \times \nabla \times \bar{E}, \\
& \nabla \times \nabla \times \bar{H}-\omega^{2} \mu \epsilon \bar{H}=(\nabla \epsilon / \epsilon) \times \nabla \times \bar{H} .
\end{aligned}
$$

Here $\bar{E}$ and $\bar{H}$ represent, respectively, the complex amplitudes of the time harmonic electric and magnetic fields. The permittivity $\epsilon(\bar{r})$ is assumed to be complex to account for conductivity, and also because in an absorbing or amplifying medium the polarization is not in phase with the electric field. Similarly the permeability $\mu(\bar{r})$ is complex to account for out-of-phase components of the magnetization. In free space $\epsilon$ and $\mu$ reduce to $\epsilon_{0}$ and $\mu_{0}$. The purpose of this section is to reduce the vector partial differential equations to a set of ordinary differential equations relevant to annular mode lasers. The most useful practical consequences of this mathematical formalism include the free space resonator modes, alignment effects, and far field patterns described in the following sections.

Equations (1) and (2) cannot be solved in general, and some restrictions are necessary. For the problem of annular gain lasers, cylindrical coordinates are the natural choice. Also, in practice Brewster windows would not normally be used, and it is convenient to consider only the cylindrical components of the vector fields. Equation (1) is really three coupled equations for the components of the electric field. If the charge density is zero and if the changes in $\epsilon$ and $\mu$ per wavelength are small, it follows from Eq. (1) that the dominant transverse field components are governed by

$$
\begin{aligned}
& \frac{1}{r} \frac{\partial}{\partial r}\left(r \frac{\partial E_{r}}{\partial r}\right)+\frac{1}{r^{2}} \frac{\partial^{2} E_{r}}{\partial \phi^{2}}+\frac{\partial^{2} E_{r}}{\partial z^{2}} \\
&+ k^{2} E_{r}=\frac{1}{r^{2}}\left(E_{r}+2 \frac{\partial E_{\phi}}{\partial \phi}\right), \\
& \frac{1}{r} \frac{\partial}{\partial r}\left(r \frac{\partial E_{\phi}}{\partial r}\right)+\frac{1}{r^{2}} \frac{\partial^{2} E_{\phi}}{\partial^{2}}+\frac{\partial^{2} E_{\phi}}{\partial z^{2}} \\
&+k^{2} E_{\phi}=\frac{1}{r^{2}}\left(E_{\phi}-2 \frac{\partial E_{r}}{\partial \phi}\right),
\end{aligned}
$$

where $k^{2}=\omega^{2} \mu \epsilon$ is the complex propagation constant. These equations can be reduced to a single scalar equation by means of the substitutions

$$
E_{r}=\mp i T_{\mp}(r, z) \exp (-i l \phi) \quad E_{\phi}=-T_{\mp}(r, z) \exp (-i l \phi) .
$$

The scalar function $T_{\mp}$ is any solution of

$$
\frac{\partial^{2} T_{\mp}}{\partial r^{2}}+\frac{1}{r} \frac{\partial T_{\mp}}{\partial r}-\frac{(l \mp 1)^{2}}{r^{2}} T_{\mp}+\frac{\partial^{2} T_{\mp}}{\partial z^{2}}+k^{2} T_{\mp}=0 .(6)
$$

The mode solutions of Eqs. (5) and (6) have helical phase fronts and are either left-hand or right-hand polarized depending on whether the upper or lower signs are chosen. Alternatively one could choose $E_{r}$ $=\mp i T_{\mp} \exp (i l \phi), E_{\phi}=T_{\mp} \exp (i l \phi)$, or for nonrotating polarization $E_{r}=\mp T_{\mp} \sin (l \phi), E_{\phi}=-T_{\mp}$ $\cos (l \phi)$.

We now postulate that the annular laser beam is localized near the radius $r_{0}$. In the vicinity of the beam the propagation constant varies so slowly that a Taylor series expansion can be used keeping only constant, linear, and quadratic terms according to $k^{2}(r, z)=k_{0}(z)\left[k_{0}(z)-k_{1}(z)\left(r-r_{0}\right)-k_{2}(z)(r-\right.$ $\left.r_{0}\right)^{2}$. Spatial variations of the propagation constant occur commonly in practice and can often have a dominant effect on beam propagation. In annular lasers leading sources of radial inhomogeneity include thermal effects, density variations, nonuniform pumping, and gain saturation. For nearly plane waves the substitution

$$
T_{\mp}=U_{\mp}(r, z) \exp \left[-i \int k_{0}(z) d z\right]
$$

reduces Eq. (6) to

$$
\begin{array}{r}
\frac{\partial^{2} U_{\mp}}{\partial r^{2}}+\frac{1}{r} \frac{\partial U_{\mp}}{\partial r}-\frac{(l \mp 1)^{2}}{r^{2}} U_{\mp}-i \frac{d k_{0}}{d z} U_{\mp}-2 i k_{0} \frac{\partial U_{\mp}}{\partial z} \\
-k_{0} k_{1}\left(r-r_{0}\right) U_{\mp}-k_{0} k_{2}\left(r-r_{0}\right)^{2} U_{\mp}=0,
\end{array}
$$

where $U_{\mp}$ has been assumed to vary so slowly with $z$ that its second derivative can be neglected. The term in $r^{-2}$ can also be expanded near $r_{0}$ according to

$$
r^{-2}=r_{0}^{-2}-2 r_{0}^{-3}\left(r-r_{0}\right)+3 r_{0}^{-4}\left(r-r_{0}\right)^{2}-\ldots .
$$

This expansion permits us to retain lowest order corrections to the propagation characteristics due to the azimuthal variations, and the results will be verified in the next section by comparison with known formulas.

The substitution

$$
U_{\mp}=V_{\mp}(r, z) \exp -i\left[Q(z)\left(r-r_{0}\right)^{2} / 2+S(z)\left(r-r_{0}\right)\right]
$$

in Eq. (8) leads to the separation 


$$
\begin{gathered}
Q^{2}+k_{0} \frac{d Q}{d z}+k_{0} k_{2}+\frac{3(l \mp 1)^{2}}{r_{0}^{4}}=0, \\
Q S+k_{0} \frac{d S}{d z}+\frac{k_{0} k_{1}}{2}-\frac{(l \mp 1)^{2}}{r_{0}^{3}}=0, \\
\frac{\partial^{2} V_{\mp}}{\partial r^{2}}-2\left[i Q\left(r-r_{0}\right)+i S\right] \frac{\partial V_{\mp}}{\partial r}-S^{2} V_{\mp}-i Q V_{\mp} \\
-\frac{(l \mp 1)^{2}}{r_{0}^{2}} V_{\mp}-2 i k_{0} \frac{\partial V_{\mp}}{\partial z}-i \frac{d k_{0}}{d z} V_{\mp}=0,
\end{gathered}
$$

where we have collected terms in equal powers of $(r$ $\left.-r_{0}\right)$. For simplicity and conditions $\left|Q_{i}\right| \gg r_{0}{ }^{-2},\left|S_{i}\right|$ $Q_{i}\left|\ll r_{0},\right| S_{r} / Q_{r} \mid \ll r_{0}$ have been consistently applied, and the terms $\mp 1$ can also be dropped. The subscripts $i$ and $r$ denote, respectively, the imaginary and real parts of these functions, and it will be seen in the following paragraphs that the conditions just given localize the beam to a region that is thin compared to $r_{0}$.

Equation (13) can be reduced further. In terms of the new variables $\rho=(i Q)^{1 / 2}\left(r-r_{0}+S / Q\right), \zeta=z$, the substitution

$$
V_{\mp}=H(\rho) \exp [-i P(z)]
$$

leads to the separation

$$
\begin{gathered}
\frac{d P}{d z}=-\frac{i(n+1 / 2) Q}{k_{0}}-\frac{S^{2}}{2 k_{0}}-\frac{i}{2 k_{0}} \frac{d k_{0}}{d z}-\frac{(l \mp 1)^{2}}{2 k_{0} r_{0}{ }^{2}}, \\
\frac{d^{2} H}{d \rho^{2}}-2 \rho \frac{d H}{d \rho}+2 n H=0,
\end{gathered}
$$

where $n$ is a separation constant. Equation (16) is the Hermite differential equation, and the useful solutions are the well known Hermite polynomials $H_{n}(\rho)$. Thus the basic mode set for an annular laser medium with radial variations of the gain and index of refraction is described by the functions

$$
\begin{aligned}
T_{\mp}=H_{n}(\rho) \cdot \exp -i\left[\int k_{0} d z+\frac{Q}{2}\left(r-r_{0}\right)^{2}\right. & \\
& \left.+S\left(r-r_{0}\right)+P\right] .
\end{aligned}
$$

It is important to consider the physical significance of the parameters that have been introduced. The real and imaginary parts of the parameter $Q$ may be written explicitly as

$$
Q=\frac{2 \pi}{\lambda R}-\frac{2 i}{w^{2}}
$$

where $R(z)$ is the radius of curvature of the phase fronts in the radial direction, and $w(z)$ is the 1/e amplitude spot size. Thus we may regard Eq. (17) as a set of Hermite-Gaussian beam modes. It also follows from Eq. (17) that the ratio $d_{a}=-S_{i} / Q_{i}$ represents the distance of the amplitude center of the beam from $r_{0}$, and $d_{p}=-S_{r} / Q_{r}$ represents the distance of the phase center of the beam from $r_{0}$. The real part of the parameter $P(z)$ is a phase correction for the propagating beam, and the imaginary part of $P(z)$ is an amplitude correction.

The basic propagation characteristics of the beam modes of an annular gain medium are governed by Eqs. (11), (12), and (15). These equations are similar in form to the equations governing the propagation of conventional Gaussian beams in ordinary complex lenslike media, and the solutions have been given. ${ }^{8}$ The essential feature of the results in this case is that the beam spot size, phase front curvature, displacement from $r_{0}$, and phase all oscillate periodically as the beam propagates through the medium. If the beam center is near a gain maximum, the oscillations are damped, and the propagation is stable. On the other hand, if the beam center is near a gain minimum, the oscillations grow in amplitude, and the propagation is unstable. In the latter case stable Gaussian resonator modes cannot be obtained without the use of additional stabilizing apertures or mirror reflectivity profiles. Well known matrix techniques are useful for the actual evaluation of the resonant modes when lenslike media are present, and the details are omitted here. The free space solutions are discussed in the following section.

The phase fronts of the modes described by Eqs. (17) and (18) would have a simple circular curvature in the radial direction except that the variable $\rho$ is complex. This complexity introduces a phase ripple in the higher order modes. In the special case that there are no radial variations of the gain, a much simpler mode set can be written. In terms of the variables $\rho=\left(-Q_{i}\right)^{1 / 2}\left(r-r_{0}+S_{i} / Q_{i}\right), \zeta=z$, the substitution given in Eq. (14) reduces Eq. (13) to

$$
\begin{gathered}
\frac{d P}{d z}=-\frac{i Q_{r}}{2 k_{0}}+\frac{(n+1 / 2) Q_{i}}{k_{0}}-\frac{S^{2}}{2 k_{0}}-\frac{i}{2 k_{0}} \frac{d k_{0}}{d z}-\frac{(l \mp 1)^{2}}{2 k_{0} r_{0}^{2}} \\
\frac{d^{2} H}{d \rho^{2}}-2 \rho \frac{d H}{d \rho}+2 n H=0 \\
\frac{\partial \rho}{\partial z}=-\frac{Q_{r}\left(r-r_{0}\right)+S_{r}}{k_{0}} \frac{\partial \rho}{\partial r} .
\end{gathered}
$$

After separating out Eq. (19), Eqs. (20) and (21) follow from the requirement that $H(\rho)$ be real. One can show that Eq. (21) is consistent with Eqs. (11) and (12) only when the gain has no radial variations. Thus the modes are again Hermite-Gaussian functions. The advantage is that now the argument of the Hermite polynomials is real, and the phase fronts are characterized by simple circular curvature.

When gain variations are negligible, the propagation constant can be replaced by the index of refraction according to $k=2 \pi n / \lambda$. Then it follows from Eqs. (11) and (12) that the displacement from $r_{0}$ of the amplitude center of the beam is governed by the ray equation

$$
\frac{d}{d z}\left(n_{0} \frac{d d_{a}}{d z}\right)=-\frac{n_{1}{ }^{\prime}}{2}-n_{2}{ }^{\prime} d_{a}
$$

where the primed indices of refraction are corrected for azimuthal variations according to

$$
n_{1}^{\prime}=n_{1}-\left(\frac{\lambda}{2 \pi}\right)^{2} \frac{2 l^{2}}{n_{0} r_{0}^{3}}
$$




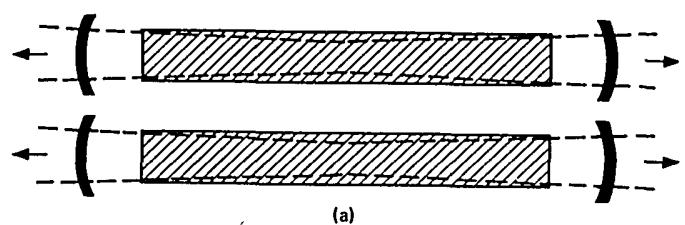

(a)

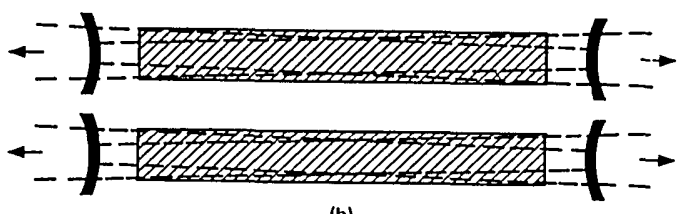

(b)

Fig. 2. Cross section through annular gain lasers showing (a) low loss and (b) high loss configurations. The high losses result from reversing the surface curvature of one or both reflectors as shown in the figure or by restricting the radial extent of the mirror annulus.

$$
n_{2}^{\prime}=n_{2}+\left(\frac{\lambda}{2 \pi}\right)^{2} \frac{3 l^{2}}{n_{0} r_{0}^{4}} .
$$

When the index of refraction is independent of $z$, the solutions of Eq. (22) are simple sinusoidal or exponential functions. The terms $\mp 1$ have been dropped here as indicated previously.

In the preceding discussion we have derived expressions for the cylindrical field components of the beam modes of annular laser media. The modes were obtained as solutions of the vector wave equations, subject to certain restrictions. These restrictions can be substantially relaxed by retaining additional terms in Eqs. (11)-(13). As more terms are kept, however, the mathematics becomes increasingly cumbersome. Fortunately, in most practical situations that one can visualize all such corrections are unimportant. Even the leading corrections involving $l$ may usually be neglected, and this point is considered further in the following section.

We conclude by deriving briefly the similar mode solutions for the Cartesian field components, which would result if a Brewster window were incorporated in the laser cavity. If the changes in $\epsilon$ and $\mu$ per wavelength are small, it follows from Eq. (1) that the $x$ or $y$ components of the electric field are governed by the single scalar equation

$$
\frac{1}{r} \frac{\partial}{\partial r}\left(r \frac{\partial E}{\partial r}\right)+\frac{1}{r^{2}} \frac{\partial^{2} E}{\partial \phi^{2}}+\frac{\partial^{2} E}{\partial z^{2}}+k^{2} E=0 .
$$

For any of the substitutions $E=T \exp (-i l \phi)$, $E=T \exp (i l \phi), E=T \cos (l \phi), E=T \sin (l \phi)$, Eq. (25) reduces to

$$
\frac{\partial^{2} T}{\partial r^{2}}+\frac{1}{r} \frac{\partial T}{\partial r}-\frac{l^{2}}{r^{2}} T+\frac{\partial^{2} T}{\partial z^{2}}+k^{2} T=0 .
$$

But this equation is identical in form to Eq. (6), and all the previous solutions are still applicable. The only difference is that the term $(l \neq 1)^{2}$ is here replaced by $l^{2}$. However, this term is only significant when $l$ is much greater than unity, so the equations are effectively identical. Therefore, the Cartesian field components may be described by either Eqs. (15) and (16) or (19) and (20), depending on whether gain profile effects are important.

\section{Free Space Resonator Modes}

In the previous section the vector wave equation has been solved for the basic propagation modes of annular gain media. The governing parameters are essentially identical to the parameters of conventional Gaussian laser beams. We now consider in greater detail the most important special case of propagation in uniform media and free space $\left(n_{1}=n_{2}=0\right)$. In some laser materials the gain and refraction profiles are too weak to have any effect on the transverse field distribution, and these results apply directly to the gas laser experiments described in the next section. Two possible resonator configurations with toric mirrors are shown in cross section in Fig. 2. The first has stable modes that are closely related to the modes of conventional lasers, and these modes will be considered in detail in the following paragraphs. The second design is a high loss resonator that would exhibit improved mode control with high gain annular laser amplifiers.

As a first example, we consider the behavior of an annular beam mode near a reference plane where the phase fronts are flat. The results are relevant for the optical fields in the vicinity of a flat resonator mirror. The solution of Eq. (22) is

$$
d_{a}(z)=\frac{n_{1}^{\prime}}{2 n_{2}^{\prime}} \cos \left[\left(\frac{n_{2}^{\prime}}{n_{0}}\right)^{1 / 2} z\right]-\frac{n_{1}^{\prime}}{2 n_{2}{ }^{\prime}} .
$$

We have only retained the leading correction terms to the propagation, and at short distances in a uniform medium $\left(n_{1}=n_{2}=0\right) \mathrm{Eq}$. (27) is

$$
d_{a}(z)=-\frac{n_{1}{ }^{\prime} z^{2}}{4 n_{0}}=\left(\frac{\lambda}{2 \pi}\right)^{2} \frac{l^{2} z^{2}}{2 n_{0}{ }^{2} r_{0}{ }^{3}} .
$$

This result implies that the effect of the azimuthal field distribution is to increase the diameter of the annular beam modes. The same conclusion follows from the qualitative notion that there is an expanding force associated with the wave that tends to spread out regions of high field amplitude. ${ }^{9}$ Similarly, from Eq. (11) one finds that the beam parameter $Q$ is governed by

$$
\frac{Q(z)}{k_{0}}=\frac{-\left(\frac{n_{2}^{\prime}}{n_{0}}\right)^{1 / 2} \sin \left[\left(\frac{n_{2}{ }^{\prime}}{n_{0}}\right)^{1 / 2} z\right]+\frac{Q(0)}{k_{0}} \cos \left[\left(\frac{n_{2}{ }^{\prime}}{n_{0}}\right)^{1 / 2} z\right]}{\cos \left[\left(\frac{n_{2}^{\prime}}{n_{0}}\right)^{1 / 2} z\right]+\frac{Q(0)}{k_{0}}\left(\frac{n_{0}}{n_{2}{ }^{\prime}}\right)^{1 / 2} \sin \left[\left(\frac{n_{2}^{\prime}}{n_{0}}\right)^{1 / 2} z\right]}
$$


where $Q(0)=-2 i / w_{0}^{2}$. In the limit of short distances this result reduces to

$$
\frac{1}{R}-\frac{i \lambda}{\pi w^{2}}=\frac{1}{z+z^{-1}\left[\frac{1}{z_{0}^{2}}-\frac{n_{2}^{\prime}}{n_{0}}\right]^{-1}}-\frac{i}{z_{0}\left[1+\left(\frac{1}{z_{0}^{2}}-\frac{n_{2}{ }^{2}}{n_{0}}\right) z^{2}\right]},
$$

where the Rayleigh length $z_{0}=\pi n_{0} w_{0}^{2} / \lambda$ has been introduced.

Equations (28) and (30) describe the propagation of thin tubular beam modes in a uniform medium including a first-order correction for azimuthal field variations. It is instructive to compare these formulas with known results. Under some circumstances the high order Laguerre-Gaussian modes of conventional spherical mirror laser resonators have a large dark region at the axis and resemble closely the modes that we have derived here. For the cylindrical field components the radial amplitude distribution of the Laguerre-Gaussian solutions to Eqs. (3) and (4) $\operatorname{are}^{8}$

$$
E \alpha\left(\frac{2 r^{2}}{w^{\prime 2}}\right)^{(l \neq 1) / 2} L_{P}^{l \neq 1}\left(\frac{2 r^{2}}{w^{\prime 2}}\right) \exp \left(-\frac{r^{2}}{w^{\prime 2}}\right)
$$

where $L_{p}^{l \neq 1}$ is the associated Laguerre polynomial, and the prime on the spot size is used to distinguish this quantity from the $1 / e$ thickness of the annular beam modes. When the index $\mathrm{p}$ is equal to zero the Laguerre polynomial reduces to $L_{0}^{l \neq 1}=(l \mp 1) !,{ }^{10}$ and for $l \gg 1$ the radial distribution is simply

$$
E \alpha\left(\frac{r^{2}}{w^{\prime 2}}\right)^{l / 2} \exp \left(-\frac{r^{2}}{w^{\prime 2}}\right) .
$$

It follows from Eq. (32) that for large $l$ the LaguerreGaussian field can be considered to be a Gaussian annulus of $1 / e$ thickness $w=w^{\prime} 2^{-1 / 2}$ centered at the radius $r=(l / 2)^{1 / 2} w^{\prime}$. In other words, the fields are a special case of the solutions we have described previously. But the propagation characteristics of the Laguerre-Gaussian modes are known exactly, so they can be used as a check on our tubular resonator modes.

The location of the amplitude center of a Laguerre-Gaussian beam must evolve in the same way as the spot size of the fundamental Gaussian mode, and the well known spot size formula leads to

$$
r_{0}+d_{a}=r_{0}\left[1+\left(\frac{\lambda z}{\pi n_{0} w_{0}{ }^{\prime 2}}\right)^{2}\right]^{1 / 2} \text {. }
$$

With the relation $w_{0}^{\prime}=(2 / l)^{1 / 2} r_{0}$, Eq. (33) reduces exactly to Eq. (28) in the limit of small $z$. Similarly, the well known Gaussian beam formulas

$$
R^{\prime}=z\left[1+\left(z_{0}^{\prime} / z\right)^{2}\right] . \quad w^{\prime}=w_{0}^{\prime}\left[1+\left(z / z_{0}^{\prime}\right)^{2}\right]^{1 / 2}
$$

are in exact agreement with Eqs. (24) and (30) when one makes the required substitutions $R=R^{\prime}, w=$ $w^{\prime} 2^{-1 / 2}, r_{0}=(l / 2)^{1 / 2} w_{0}{ }^{\prime}$, and $z_{0}{ }^{\prime}=\pi n_{0} w_{0}{ }^{2} 2 / \lambda$. Thus, very high order $(l \gg 1)$ Laguerre-Gaussian modes are a consistent special case of the annular modes that we are considering.

From the preceding discussion it is clear that con- ventional spherical mirror resonators are a possible choice for lasers in which the axial region is obscured, and in fact they have been employed previously. ${ }^{7}$ It should be emphasized, however, that the resulting Laguerre-Gaussian modes have a very serious deficiency in comparison to the tubular modes that we have derived. The only Laguerre-Gaussian modes that have a small amplitude near the axis are those with very large values of either the mode index $l$ or $p$. For example, for the tubular modes described by Eq. (32) the ratio of the spot size to the radius of maximum amplitude is equal to $l^{-1 / 2}$. Thus a value $l=$ 100 would be required to obtain a ratio $w / r=0.1$. The trouble with such high order modes is that they have a large number of node lines across their phase fronts, and they exhibit very rapid diffraction. One can readily show that the far field diffraction angle is $\theta_{\text {diff. }}=r / z=l \lambda\left(2 \pi r_{0}\right)^{-1}$, i.e., $l / 2$ times larger than a conventional Gaussian beam with a spot size equal to $r_{0}$. Furthermore, the ratio $w / r$ is independent of $z$ so no energy density will be near the center of a distant target. By contrast, the annular modes we derived previously can have a large hole near the axis without having any node lines across the phase front.

We now indicate briefly how the far field emission pattern of the annular gain lasers may be calculated. From conventional vector diffraction theory, one finds that the electric field distribution $\bar{E}_{2}$ at a distant surface is related to the field $\bar{E}_{1}$ at an input surface by the equation ${ }^{11}$

$$
\bar{E}_{2}=\frac{1}{4 \pi} \int_{s} \int\left(\bar{E}_{1} \frac{\partial \gamma}{\partial n}-\gamma \frac{\partial \bar{E}_{1}}{\partial n}\right) d s,
$$

where the function $\gamma$ is given by $\gamma=\exp (-i k|\bar{r}|) / \bar{r} \mid$, $\bar{n}$ is the normal to the input surface in the direction of propagation, and the integral is over the input. The vector $\bar{r}$ connects points on the input and output surfaces. A similar result applies to the magnetic fields. For a plane input surface and paraxial waves having transverse field variations that are slow compared to a wavelength, Eq. (35) reduces to

$$
\bar{E}_{2}=\frac{i}{\lambda} \int_{s} \int_{\bar{E}_{1}} \frac{\exp (-i k|\bar{r}|)}{|\bar{r}|} d s .
$$

This is the vector equivalent of the familiar Huygen's principle. ${ }^{12}$ If the second surface is a plane parallel to the input surface and separated from it by a distance $L$, the vector $\bar{r}$ can be written

$$
|\bar{r}|=\left[L^{2}+r^{2}+r^{\prime 2}-2 r r^{\prime} \cos \left(\phi-\phi^{\prime}\right)\right]^{1 / 2},
$$

where $r$ and $\phi$ are polar coordinates on the input plane, $r^{\prime}$ and $\phi^{\prime}$ are polar coordinates on the output plane, and the law of cosines has been employed. In the far field $(r \ll L)$ Eqs. (36) and (37) yield 


$$
\begin{aligned}
\bar{E}_{2}=\frac{i \exp \left[-i k\left(L+r^{\prime 2} / 2 L\right)\right]}{\lambda L} \int_{0}^{2 \pi} \int_{0}^{\infty} \bar{E}_{1} \\
\quad \times \exp \left[\frac{i k r r^{\prime}}{L} \cos \left(\phi-\phi^{\prime}\right)\right] r d r d \phi .
\end{aligned}
$$

As an example, we assume that the input is a linearly polarized plane wave beam mode in the form

$$
\bar{E}_{1}=\bar{i}_{x} f\left(r-r_{0}\right) \cos (l \phi) .
$$

This is a possible form for an annular beam of radius $r_{0}$ emerging through a plane laser mirror. The $\phi$ integration in Eq. (38) can be performed resulting in the spherical wave ${ }^{13}$

$$
\begin{aligned}
\bar{E}_{2}=\frac{\bar{i}_{x} 2 \pi i^{l+1} \exp \left[-i k\left(L+r^{\prime 2} / 2 L\right)\right] \cos \left(l \phi^{\prime}\right)}{\lambda L} \\
\times \int_{0}^{\infty} f\left(r-r_{0}\right) J_{l}\left(\frac{k r \gamma^{\prime}}{L}\right) r d r .
\end{aligned}
$$

For large values of $L$ the Bessel function varies slowly with $r$ in comparison to $f\left(r-r_{0}\right)$, and Eq. (40) is simply

$$
\begin{array}{r}
\bar{E}_{2}=\bar{i}_{x} \frac{2 \pi i^{l+1} \exp \left[-i k\left(L+\gamma^{2} / 2 L\right)\right] \cos \left(l \phi^{\prime}\right)}{\lambda L} J_{l}\left(\frac{k r_{0} \gamma^{\prime}}{L}\right) \\
\times \int_{0}^{\infty} f\left(r-\cdot r_{0}\right) r d r .
\end{array}
$$

For $l=0$ we may define the diffraction angle $\theta_{\text {diff }}$ as the angle to the first zero of $J_{0}$ or

$$
\theta_{\mathrm{diff}}=\frac{r^{\prime}}{L}=\frac{2.405 \lambda}{2 \pi r_{0}}=0.383 \frac{\lambda}{r_{0}^{\prime}},
$$

which is about the same as the diffraction angle for a slit of width $2 r_{0}$. On the other hand, it was indicated previously that the diffraction angle of a high order Laguerre-Gaussian mode of radius $r_{0}$ has the much larger value $\theta_{\text {diff }}=l \lambda\left(2 \pi r_{0}\right)^{-1}$. Thus for resonators having the axial region obscured, the annular resonators described here provide a substantial improvement in far field beam collimation.

The actual evaluation of the resonant modes of a laser oscillator follows along standard lines. The re- quirement that after one round trip the fields must reproduce themselves in both amplitude and phase reduces to the requirement that the complex parameters $Q$ and $S$ repeat and that the phase delay is an integral multiple of $2 \pi$. One finds from Eq. (30) that for most practical lasers the terms involving the azimuthal mode index $l$ can be neglected, and the analysis simplifies further. Then the free space beam modes are

$$
\begin{aligned}
& T_{\mp}=\left(\frac{w_{0}}{w}\right)^{1 / 2} H_{n}\left[\frac{2^{1 / 2}\left(r-r_{0}\right)}{w}\right] \exp -i\left[k_{0} z\right. \\
& \left.+\frac{k_{0}\left(r-r_{0}\right)^{2}}{2 R}-i \frac{\left(r-\gamma_{0}\right)^{2}}{w^{2}}-\left(n+\frac{1}{2}\right) \tan ^{-1} \frac{z}{z_{0}}\right],
\end{aligned}
$$

where the amplitude factor $\left(w_{0} / w\right)^{1 / 2}$ and the $\tan ^{-1}$ phase factor result from solving Eq. (19) with $S=0$ and $(l \mp 1)^{2} \simeq 0$. This simplified expression is identical with the original annular resonator modes that were derived by another method, ${ }^{3}$ and an example of the evaluation of $R$ and $w$ is given in the following section.

\section{Experiment}

Several experiments have been performed to verify the theoretical properties of annual gain resonators. TEA CO ${ }_{2}$ discharges are a natural choice, and one experimental configuration is shown in Fig. 3. The cathode is a 1-in. $(2.54-\mathrm{cm})$ diam aluminum rod into which is cut a thin spiral groove. A plastic insulated trigger wire (Belden 8868) enters along the axis of the cathode rod and is wound into the machined groove. The anode is a $7.6-\mathrm{cm}$ i.d. aluminum tube of $76-\mathrm{cm}$ length, and the length of the active discharge region is $64 \mathrm{~cm}$. The left-hand end of the cathode rod is supported by a machined and polished aluminum laser mirror having its center of curvature at a radius of $r_{0}=2.5 \mathrm{~cm}$. The cathode rod is insulated from the mirror by means of a Plexiglas sleeve, and all mechanical joints are vacuum sealed by o rings. The right-hand end of the cathode rod is attached to a short Plexiglas rod, which is supported by four thin adjustable steel pins. These pins permit precise cen-

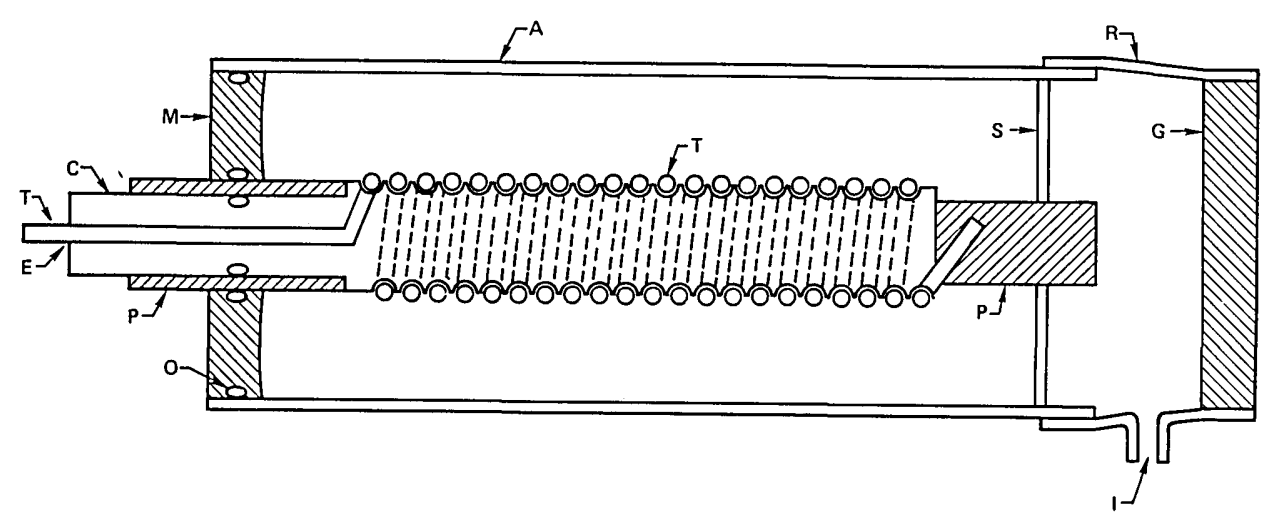

Fig. 3. Cross sectional drawing of a coaxial $\mathrm{CO}_{2}$ laser showing $A$, aluminum anode pipe; $C$, machined aluminum cathode rod; $M$, machined and polished aluminum mirror; $S$, steel support pins; $P$, Plexiglas insulators; $T$, trigger wire; $O$, o rings; $R$, rubber diaphragm; $I$, gas inlet; and $E$, gas exhaust around trigger wire.

Laser length is not to scale. 


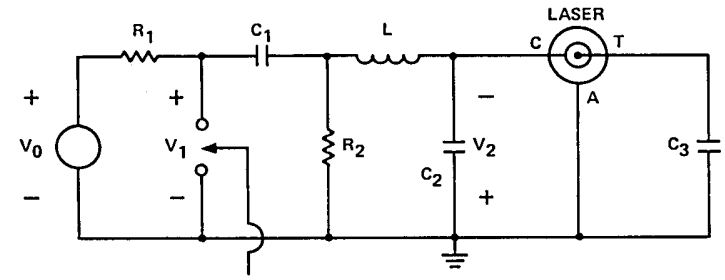

Fig. 4. Electrical circuitry for the coaxial double-discharge laser. The laser is shown schematically in cross section with the notation: $A$, anode; $C$, cathode; $T$, trigger.

tering of the cathode without introducing excessive optical loss. The right-hand germanium mirror is gimbal-mounted and attached to the laser anode by means of a rubber diaphragm. Some of our other designs have been sealed at the right-hand mirror for operation below or above atmospheric pressure. So far we have found little advantage in such designs, however, and our best results were obtained with the device shown in the figure. A mixture of carbon dioxide, helium, and nitrogen $(5 \%, 90 \%, 5 \%)$ enters through the diaphragm and is allowed to exit around the trigger wire.

The electrical discharge circuit is shown in Fig. 4 and is similar in many respects to the circuitry used with previous double-discharge devices. ${ }^{14-16}$ The basic objective is to develop a corona-ionized region near the cathode prior to the main laser discharge. This preionization serves to delay the onset of arc formation until after the main discharge and the laser pulse. The capacitor $C_{1}=0.25 \mu \mathrm{F}$ is first charged through the resistance $R_{1}=5 \mathrm{M} \Omega$ and $R_{2}=$ $50 \Omega$ to a voltage $V_{1}$ of between $15 \mathrm{kV}$ and $25 \mathrm{kV}$. When the spark gap is triggered, the voltage across the laser is determined initially by the combination of $C_{1}, C_{2}$, and $L$ according to

$$
V_{2}(t)=\frac{V_{1}(0)}{1+C_{2} / C_{1}}(1-\cos \omega t) .
$$

The capacitance $C_{3}=0.002 \mu \mathrm{F}$ is too small for it to affect the oscillation characteristics of the circuit. The resistor $R_{2}$ provides some damping of the oscillations, but for our circuit values $R_{2}$ has a negligible effect on the initial pulse rise time. From Eq. (44) the time delay of the first voltage maximum is

$$
t_{d}=\frac{\pi}{\omega}=\pi\left[\frac{1}{L}\left(\frac{1}{C_{1}}+\frac{1}{C_{2}}\right)\right]^{-1 / 2},
$$

and the peak value is

$$
V_{2 \max }=\frac{2 V_{1}(0)}{1+C_{2} / C_{1}} .
$$

We have tried a range of component values in our experiments, but best results were obtained with the values indicated previously together with $L=15 \mu \mathrm{H}$ and $C_{2}=0.05 \mu \mathrm{F}$. From Eqs. (45) and (46) the pulse delay is $t_{d}=2.5 \mu \mathrm{sec}$, and the peak voltage is $V_{2 \max }$ $=1.7 V_{1}(0)$.
The basic operation of the discharge circuit is as follows. During the risetime of the negative voltage pulse, a corona current flows charging the capacitance $C_{3}$ and creating a region of ionization close to the cathode. Near the peak of the voltage pulse the main discharge begins, and most of the energy in $C_{2}$ is used. The remaining energy in the circuit feeds the tail of the laser pulse, and with proper adjustment the voltage remains low enough that arcing usually does not occur.

The laser resonator consists of two mirrors spaced by $0.8 \mathrm{~m}$. The output mirror is a gimbal mounted germanium flat that has a reflectivity of $80 \%$, and the back surface is antireflection coated. The second mirror is machined to the shape shown in cross section in Fig. 3 with a radius of curvature of $2 \mathrm{~m}$ centered at a radius of $2.5 \times 10^{-2} \mathrm{~m}$. As indicated in the previous section, the modes in a free space laser of these dimensions are governed to a good approximation by the well known mode formulas for a conventional laser. For our half-symmetric device the spot size of the fundamental mode at the flat mirror is

$$
w_{0}=(\lambda / \pi)^{1 / 2}[d(R-d)]^{1 / 4},
$$

while the spot size at the curved mirror is

$$
w_{1}=(\lambda d R / \pi)^{1 / 2}[d(R-d)]^{-1 / 4} .
$$

The free space approximation is valid for our work because the low density laser plasma causes a negligible refraction of the $10.6-\mu \mathrm{m}$ laser beam. Deflection of the beam by the gain profile can sometimes be important in high gain $\mathrm{CO}_{2}$ lasers, but this effect is also negligible in the present study. ${ }^{17}$

We have viewed the output transverse mode behavior of our lasers using thermographic phosphors and liquid crystal films. The fundamental property of the output is that the radiation is confined to a thin annular region at a radius of approximately 2.5 $\times 10^{-2} \mathrm{~m}$. Lasing is uniform about the axis, and at low pumping levels the radial variation of intensity is well represented by a Gaussian of spot size $w_{0} \simeq 2$ $\mathrm{mm}$ in agreement with Eq. (47). The small number of high order radial node lines is the principal advantage of this type of resonator, and measurements made at distances up to several meters from the laser show the expected symmetric thickening of the sides of the annular modes. At higher pumping levels the output is in the form of concentric rings, indicating operation in Hermite-Gaussian radial modes up to order $n=3$. All evidence so far indicates that the mode formalism of the previous section provides an excellent description of the experimental results.

Slight misalignment of the laser mirrors has a strong effect on the output beam. When misalignment occurs, the radiation becomes localized at the side of the annulus where the cavity length is greatest. This effect can be understood theoretically by recalling that in our analysis the radial and azimuthal variations are almost completely decoupled. Then tilting the mirror yields the equivalent of a long ellipsoidal mirror, i.e., confining quadratic phase varia- 


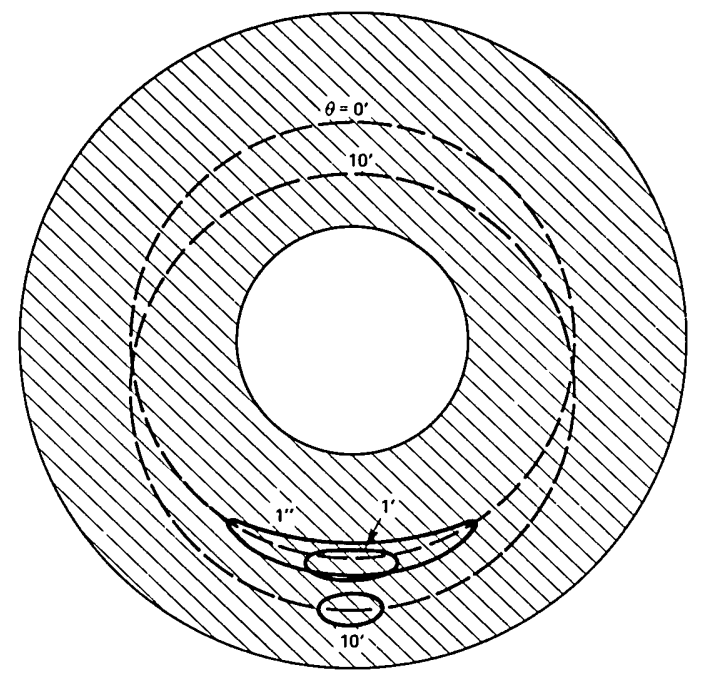

Fig. 5. Fundamental mode emission patterns for an annular gain laser misaligned as indicated. The dashed lines show the amplitude centers of higher order azimuthal modes.

tions in the $r$ and $\phi$ directions about the location of greatest cavity length. If the mirror is tilted by an angle $\theta$ about its diameter, one readily finds that the transverse variations of the mirror phase are governed by the factor

$$
\exp -i \cdot\left[\frac{k_{0}}{2 R}\left(r-r_{0}\right)^{2}-k_{0} r \tan \theta \cos \phi\right],
$$

where $R$ is the radial phase curvature of the mirror, and the azimuthal angle $\phi$ is measured from the side of greatest cavity length. From Eq. (49) it follows that the amplitude center of the beam is located at the radius

$$
r_{a}=r_{0}+R \tan \theta \cos \phi,
$$

and for $\phi \lesssim 1$ the effective phase curvature of the mirror in the $\phi$ direction is

$$
R_{\phi}=r_{0} \cot \theta .
$$

The spot size in the $\phi$ direction is now given by Eq. (47), and the over-all amplitude surfaces can also be readily determined.

The 1/e amplitude curves of the fundamental mode at the output mirror surface are plotted in Fig. 5 for values appropriate to our experiment $(R=2 \mathrm{~m}$, $d=0.8 \mathrm{~m})$. The dashed lines in the figure are plotted directly from Eq. (50) and show the amplitude centers of the higher order azimuthal modes of the misaligned resonator. Our experimental observations near threshold are in excellent agreement with Fig. 5 including the elongation of the modes as alignment is approached and the radial shift of the amplitude center. For a uniform fundamental ring mode the mirrors would have to be parallel to within about $1 \mathrm{sec}$ of arc. In practice, however, nonfundamental ring modes with node lines are readily obtained when the mirrors are misaligned by as much as a few min of arc. Improved mode control could be obtained with high loss resonators and beam compacting techniques.

The output power characteristics of our laser are essentially identical to those of more conventional double-discharge lasers. Typical output curves are shown in Fig. 6. With increased pumping the peak power increases, and the pulse delay is reduced in qualitative agreement with the relationship $P_{p} \alpha\left(t_{p}\right.$ $\left.-t_{0}\right)^{-2}$, where $P_{p}$ is the peak output power, and $t_{p}$ is the time at the output maximum. ${ }^{4}$ Far above threshold there is a tail on the output pulse due either to continued pumping or energy stored in the $N_{2}$ molecules. The measurements were made using a fast photon drag detector operating into a $50-\Omega$ load. The peak output power that we have observed for this laser is approximately $15 \mathrm{MW}$ with an applied voltage of $V_{1}=25 \mathrm{kV}$.

\section{v. Conclusion}

In this work a new class of laser resonators has been considered in detail. The distinctive feature of these resonators is that the resonant modes are localized in an annular region of space. The modes are well suited to new types of laser amplifiers in which the gain region inherently is annular in shape. Examples include certain dye and solid lasers as well as chemical and transverse-electrical gas lasers. Our experimental studies have centered on coaxial $\mathrm{CO}_{2}$ TEA devices. The observed mode characteristics are in good agreement with the theoretical predictions, and further investigations are in progress regarding techniques for discriminating against higher order azimuthal modes. Besides the design shown in Fig. 3 , we have also tried the inverse geometry in which the central rod is the anode, and the aluminum pipe is the cathode. In this case preionization is achieved by having the trigger wire spiral around the inside surface of the cathode pipe between the turns of a specially designed slinky spring. ${ }^{18}$ Resistive-pin and uv-preionized coaxial lasers are also possible. The basic mode characteristics of all such annular gain lasers can be understood in terms of familiar beam pa-

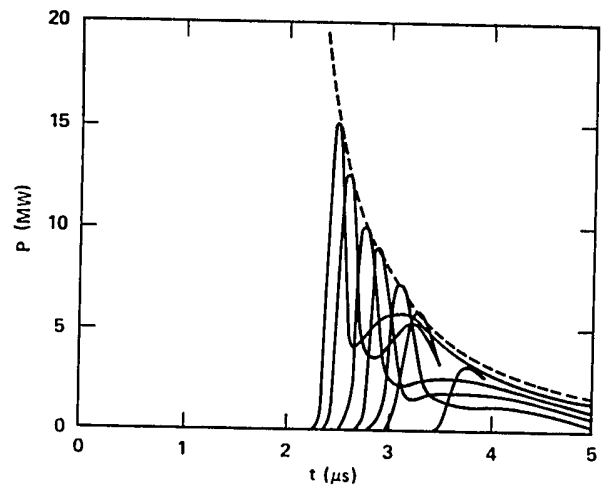

Fig. 6. Output power vs time for a coaxial double-discharge $\mathrm{CO}_{2}$ laser with various input energies. The power supply voltage increases in kilovolt steps from $V_{1}=19 \mathrm{kV}$ to $V_{1}=25 \mathrm{kV}$. 
rameter equations. With annular resonator designs, one obtains a new degree of freedom in the development of compact laser oscillators.

The authors acknowledge valuable discussions with M. E. Marhic and O. M. Stafsudd.

\section{References}

1. G. Goubau and F. Schwering, IRE Trans. Antennas Propag. AP-9, 248 (1961).

2. G. D. Boyd and J. P. Gordon, Bell Syst. Tech. J. 40, 489 (1961).

3. L. W. Casperson, J. Opt. Soc. Am. 63, 25 (1973).

4. L. W. Casperson and C. Romero, IEEE J. Quantum Electron. QE-9, 484 (1973).

5. P. Burlamacchi, R. Pratesi, and L. Ronchi, Appl. Opt. 14, 79 (1975).

6. D. Milam and H. Schlossberg, J. Appl. Phys. 44, 2297 (1973).

7. R. K. Garnsworthy, L. E. S. Mathias, and C. H. H. Carmichael, Appl. Phys. Lett. 19, 506 (1971).

8. L. W. Casperson, Appl. Opt. 12, 2434 (1973).
9. P. K. Tien, J. P. Gordon, and J. R. Whinnery, Proc. IEEE 53, 129 (1965).

10. P. M. Morse and H. Feshbach, Methods of Theoretical Physics (McGraw-Hill, New York, 1953), p. 784.

11. J. A. Stratton, Electromagnetic Theory (McGraw-Hill, New York, 1941), p. 469.

12. M. Born and E. Wolf, Principles of Optics (Pergamon, New York, 1970), p. 375.

13. I. S. Gradshteyn and I. M. Ryzhik, Table of Integrals, Series, and Products (Academic, New York, 1965), p. 482.

14. A. K. Laflamme, Rev. Sci. Instrum. 41, 1578 (1970).

15. Y.-L. Pan, A. F. Bernhardt, and J. R. Simpson, Rev. Sci. Instrum. 43, 662 (1972).

16. G. Girard, M. Huguet, and M. Michon, IEEE J. Quantum Electron. QE-9, 426 (1973).

17. L. W. Casperson and S. J. Sheldrake, Opt. Commun. 12, 349 (1974).

18. Manufactured by James Industries, Inc., Hollidaysburg, Pennsylvania.

\author{
IEEE REGION V ANNUAL CONFERENCE \\ "Electrical Engineering for this Decade" \\ Aprit 14-16, 1976 \\ Joe C. Thompson Conference Center \\ The University of Texas at Austin \\ Austin, Texas 78712
}

SCOPE: The Conference will provide a forum for technical developments in all areas of Electrical Engineering. Tutorial, survey, and research papers are solicited in any of four broad areas:

1. Antennas/EM Waves/Acoustics

2. Computers/Information Systems/Biomedical Electronics

3. Energy Resources/Energy Processing/Environmental Engineering

4. Solid State Electronics/Quantum Electronics/Networks and Systems

One technical session will feature speakers in the IEEE Student Papers Contest.

INFORMATION FOR AUTHORS: Authors are requested to submit a 50 word abstract and a detailed summary including references and figures, in total not more than 3 typewritten pages, by November 15, 1975 to:

Program Chairman: A. Bruce. Buckman

Electrical Engineering Department

The University of Texas at Austin

Austin, Texas 78712

PROCEEDINGS: Abstracts and complete accepted papers will appear in the Conference Proceedings which will be available at the Conference. 